\title{
Reversible Posterior Leukoencephalopathy Syndrome. Is It Reversible and thus Benign?
}

\author{
Key words: hypertensive encephalopathy, eclampsia, angiitis, angiopathy, angiospasm
}

Since Hinchey et al (1) described the syndrome of posterior reversible leukoencephalopathy (RPLE) in 1996, several reports have appeared in the literature. Most reports discussed similar observations in different disease settings or detailed neuroimaging observations. In the current issue of Internal Medicine, two interesting reports on this particular syndrome are included $(2,3)$.

RPLE has often been associated with diverse, mostly systemic disorders that include hypertensive encephalopathy, eclampsia, and the use of cyclosporine A, tacrolimus, interferon alpha, and erythropoietin. Transfusion-related occurrence of this syndrome has also been described with angiographic evidence of vasoconstriction (4). In this issue, Tajima et al (2) reported this syndrome in two previously healthy young women.

See also p 54.

Their neuroimaging studies disclosed arteriopathic changes confined to the posterior cerebral artery. The report illustrates a similar, if not identical disorder to RPLE having occurred without apparent underlying diseases. Thus, some unknown angiopathic processes may give rise to a similar clinical picture. Migraine and the use of oral contraceptive remain possibilities. The autopsy report by Obhu et al (3) is of importance in providing proof of the reversibility of this syndrome.

See also $\mathrm{p} 73$.

A patient with progressive systemic sclerosis developed RPLE in the setting of respiratory decompensation due to pulmonary fibrosis. Although she recovered from RPLE, she subsequently died of intestinal perforation. The autopsy revealed no cerebral pathology to account for RPLE except for some patchy gliotic changes.

However, there are two questions that are to be answered. One is if the syndrome is truly reversible. The question reminds me of a case I encountered many years ago (5). A woman in hyperemesis gravidarum developed cortical blindness and agitated delirium. Although improved, she remained severely disabled due to persistently constricted vision and mental changes. Technetium brain scanning, which was the only neuroimaging tool available at the time, disclosed prominent bilateral posterior isotope uptake for a prolonged period of time.
Also, my recent experience (unreported) of a woman with thyrotoxic crisis was a regrettable one. She did develop, what was considered to be RPLE by computed tomography (CT), following a bout of convulsions, but she died several days later without regaining consciousness. Although the reversibility has been emphasized in this particular syndrome, it will be important to promptly recognize the offending drugs and deranged metabolic state or hypertension, if any, and to treat the problem accordingly. Otherwise, it is likely that one may remain with residual neuropathological and clinical deficits. In fact, there likely are cases of "irreversible" posterior encephalopathy, although not often reported $(6,7)$.

The second question to be answered is whether or not the process is restricted to the white matter (leukoencephalopathy). Several subsequent reports in fact contain magnetic resonance (MR) imaging abnormalities beyond the white matter in the cortical gray. It is conceivable that, if angiospasm or angiopathy together with vascular leakage process is responsible for the syndrome, the cortical gray matter may not be an exception, despite the fact that vasogenic edema is more prone to occur in the white matter.

Although there are reports demonstrating similar clinical pictures with different offending settings, some other considerations are necessary to totally understand RPLE. Several reports $(2,4)$ have shown apparent arteriopathic or angiospastic processes in the posterior cerebral artery. The recent case report of postpartum cerebral angiopathy by Sugiyama et al (8) may illuminate the other side of the problem. Accumulated evidence indicates that isolated angiitis of the central nervous system (CNS), a vasculitic disease with an unfavorable prognosis, may not be unfavorable, when it occurs in puerperium. Indeed, benign postpartum angiopathy may not be posterior dominant and involve multiple intracranial arteries with varying ischemic complications (9). Both RPLE and benign angiopathy are often associated with pregnancy or puerperium, suggesting that they may share a similar pathogenesis.

It is my opinion that the reversibility and the localization to the white matter may be related to the magnitude and duration of the underlying process. Although the syndrome of RPLE may represent a preferential involvement of the posterior cerebral hemispheres, the term "reversible leukoencephalopathy" should be used with caution. Otherwise, one may be mislead by the term and the prompt treatments necessary for "reversal" of 
the syndrome may not be initiated. This point was in fact emphasized in the original report (1). More appropriate terms to describe this particular syndrome are desirable.

Teiji Yamamoto, MD The Department of Neurology, Fukushima Medical University, Hikarigaoka 1, Fukushima 960-8164

\section{References}

1) Hinchey J, Chaves C, Appignani B, et al. A reversible posterior leukoencephalopathy syndrome. New Engl J Med 334: 494-500, 1996.

2) Tajima Y, Isonishi K, Kashiwaba T, Tashiro K. Two similar cases of encephalopathy, possibly a reversible posterior leukoencephalopathy syndrome: Serial findings of magnetic resonance imaging. SPECT and angiography. Intern Med 38: 54-58, 1999.

3) Ohbu S, Uekusa T, Watanabe F, Chohnabayashi N. Histologic evidence of reversible posterior leukoencephalopathy syndrome. Intern Med 38: 73, 1999.
4) Ito $Y$, Niwa $H$, Iida $T$, et al. Post-transfusion reversible posterior leukoencephalopathy syndrome with cerebral vasoconstriction. Neurology 49: 1174-1175, 1997.

5) Yamamoto T, Suematsu K, Kano K, Hotta T, Tsuchida H. A case of bioccipital infarction following toxemia of pregnancy. Consideration of its unique brain scintigraphy in relation to the vascular anatomy of the striate cortex. Clin Neurol (Tokyo) 17: 384-390, 1977 (in Japanese with English abstract).

6) Williams EJ, Oatridge A, Holdcroft A, Goldman JM, Bydder GM. Posterior leukoencephalopathy syndrome. Lancet 347: 1556-1557, 1996.

7) Pullicino P, Zimmer W, Kwen PL. Posterior leukoencephalopathy syndrome. Lancet 347: 1557, 1996 (letter).

8) Sugiyama $Y$, Muroi A, Ishikawa $M$, Tsukamoto $T$, Yamamoto T. A benign form of isolated angitis of the central nervous system: An identical disorder to postpartum cerebral angiopathy. Intern Med 36: 931-934, 1997.

9) Calabrese LH, Graggs LA, Furlan AJ. Benign angiopathy; a distinct subset of angiographically defined primary angiitis of the central nervous system. J Rheumatol 20: 2046-2050, 1993. 